\title{
A pilot study for development of a novel tool for clinical decision making to identify fallers among ophthalmic patients
}

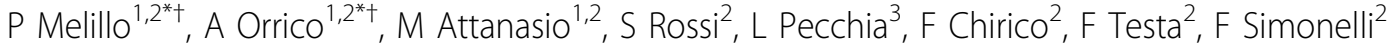

\begin{abstract}
Background: Falls in the elderly is a major problem. Although falls have a multifactorial etiology, a commonly cited cause of falls in older people is poor vision. This study proposes a method to discriminate fallers and nonfallers among ophthalmic patients, based on data-mining algorithms applied to health and socio-demographic information.

Methods: A group of 150 subjects aged 55 years and older, recruited at the Eye Clinic of the Second University of Naples, underwent a baseline ophthalmic examination and a standardized questionnaire, including lifestyles, general health, social engagement and eyesight problems. A subject who reported at least one fall within one year was considered as faller, otherwise as non-faller. Different tree-based data-mining algorithms (i.e., C4.5, Adaboost and Random Forest) were used to develop automatic classifiers and their performances were evaluated by assessing the receiver-operator characteristics curve estimated with the 10-fold-crossvalidation approach.

Results: The best predictive model, based on Random Forest, enabled to identify fallers with a sensitivity and specificity rate of $72.6 \%$ and $77.9 \%$, respectively. The most informative variables were: intraocular pressure, best corrected visual acuity and the answers to the total difficulty score of the Activities of Daily Vision Scale (a questionnaire for the measurement of visual disability).

Conclusions: The current study confirmed that some ophthalmic features (i.e. cataract surgery, lower intraocular pressure values) could be associated with a lower fall risk among visually impaired subjects. Finally, automatic analysis of a combination of visual function parameters (either self-evaluated either by ophthalmological tests) and other health information, by data-mining algorithms, could be a feasible tool for identifying fallers among ophthalmic patients.
\end{abstract}

\section{Background}

Falls represent a major problem for modern societies given its burden and implication on quality of life and autonomy of elderly and their informal caretakers [1]. The mean and median costs for a fall are about 9,000 and 11,000 euro [2]. Falls are caused by complex and dynamic interactions between intrinsic (subject-based) and extrinsic (environmental) factors [3]. Although over 400 risk factors have been identified [4] and their prioritization remains unclear [5], a commonly cited cause of

\footnotetext{
*Correspondence: paolo.melillo@unina2.it; ada_orrico@hotmail.it

+ Contributed equally

'SHARE Project, Italian Ministry of Education, Scientific Research and University, Rome, Italy

Full list of author information is available at the end of the article
}

falls in older people is poor vision. In this regard, several population-based studies have identified poor vision as one of the most frequent risk factors for falls [6-9].

Compared with normal-sighted persons, individuals with visual impairment are almost twice as likely to fall and to have recurrent falls[10]. However, the applicability, sensitivity and particularly, the specificity of subject-specific assessment of falls' risks remain imprecise[11]. For example, several functional mobility tests were proposed in literature to identify subjects at higher risk of falls and their performances were tested and compared showing that none of the test achieved an excellent predictive accuracy for the assessment of falls risk in older people[12]. This could be explained by the fact that the causes of falls are 
multifactorial with several unrelated to mobility, e.g., poor vision, cardiovascular conditions.

The current paper proposes a novel tool to identify fallers among ophthalmic patients by using data-mining methods applied to vision assessment and questionnaire to achieve information about participants' lifestyle, eye symptoms, use of glasses, systemic medical and ocular surgical history, and current medications.

\section{Methods}

\section{Study population and ethical approval}

This study was conducted on a group of subjects aged 55 years and over, enrolled among the patients visited at the Eye Clinic of the Second University of Naples from February to July 2014. The research followed the tenets of the Declaration of Helsinki and each subject gave informed consent to participate in the study. Ethics approval was obtained from the Institutional Review Board of the Second University of Naples.

Socio-demographic and medical data were recorded with a standardized questionnaire that was developed $a d$ hoc, including the variables summarized in Table 1 Table 2 and Table 3. Selected variables, which have been considered in previous studies investigating risk factors for falls, included but were not limited to those on visual impaired subjects [13-23]. In particular, information about lifestyle (e.g., cigarette smoking habit, alcohol consumption, job activity, social engagement, etc), systemic medical history (e.g., history of cancer), and general physical health (e.g. sleep problems, walking aid use, depression, hypertension, diabetes, urinary incontinence, arthritis, Parkinson disease, number and type of prescribed drugs, etc) were recorded. Moreover, a global rating of subjective health was also assessed.

All participants attended a baseline assessment where they underwent an eye examination, including assessment of the presence and severity of lens opacities, test of the best-corrected visual acuity (BCVA) by Snellen chart and measurement of the intraocular pressure (IOP). IOP was measured by using Goldmann applanation tonometry and in case of IOP than higher than $20 \mathrm{mmHg}$ the ocular medical records of the patients were reviewed in order to find previous diagnosis of glaucoma, slit-lamp biomicroscopy of anterior segment, fundus examination and computerized visual field were performed to assess if the optic nerve is damaged in order to pose diagnosis of glaucoma. Moreover, if assessed by medical record, recent worsening in the visual acuity or change in the manifested refraction were recorded. Finally, vision problems (e.g. blinding effect when exit from indoor environment, or when entering indoor environment), use of multifocal glasses and of eye drops were asked to each participant.

Visual disability was assessed by the Activity of Daily Vision Scale (ADVS) in the 15-item version proposed by Pesudovs et al.[24]. For each item, the patient was asked whether if he/she engaged in the activity (if not it is "Not Applicable" which is treated as missing data), and then the level of difficulties in doing the activity: no difficulty (5), a little difficulty (4), moderate difficulty (3), extreme difficulty (2), unable to perform the activity because of poor vision (1). Finally, the average score for the 15 items was computed.

A fall was defined as unintentionally coming to the ground or some lower level not as a result of a major

Table 1 Socio-demographic information assessed with the structured questionnaire.

\begin{tabular}{|c|c|c|}
\hline Variables & Categories or unit of measures & References \\
\hline Gender & male; female & {$[15-17,21]$} \\
\hline Age & Years & \\
\hline Indipendent life & Yes; no & [21] \\
\hline Health compared with that of age group & $\begin{array}{l}\text { Much more healthy; More healthy; About as healthy; } \\
\text { Less healthy; Much less healthy }\end{array}$ & [15] \\
\hline Living alone & Yes; no & [21] \\
\hline Type of house & Condominium; single apartment & [21] \\
\hline Jobs & merchant or craftsman; worker; employed; freelancer; other & [21] \\
\hline Retired & Yes; no & {$[13,21]$} \\
\hline Frequency pushing/dragging heavy loads & Never; Occasionally; 1-2 per week; Daily; Several times per day & [14] \\
\hline Attendance at religious service in previous month & Yes; no & [14] \\
\hline Attendance at club meeting in previous month & Yes; no & [14] \\
\hline Owns or cares for a pet & Yes; no & [14] \\
\hline Sufficient contact with family/friends & Sufficient; insufficient & [14] \\
\hline Ability to raise $€ 350$ in an emergency & $\begin{array}{l}\text { No difficulty; A little difficulty; Lot of difficulty } \\
\text { Impossible to raise } € 350\end{array}$ & [14] \\
\hline
\end{tabular}


Table 2 Medical history information assessed with the structured questionnaire.

\begin{tabular}{|c|c|c|}
\hline Variables & Categories or unit of measures & References \\
\hline Weight & $\mathrm{Kg}$ & {$[15,16]$} \\
\hline Body mass index & $\mathrm{Kg} / \mathrm{m}^{2}$ & {$[17,18,21]$} \\
\hline Smoking habit & yes; no; ex & {$[15-17,21]$} \\
\hline Alcohol consumption & Never; occasionally; usually & {$[15,21]$} \\
\hline Depression & Yes; no & {$[21,22]$} \\
\hline Anxiety & Yes; no & {$[17]$} \\
\hline Urinary incontinency & Yes; no & {$[21,22]$} \\
\hline Osteoarthitis & Yes; no & {$[16,21,22]$} \\
\hline Hypertension & Yes; no & {$[17]$} \\
\hline Diabetes & Yes; no & {$[16-18]$} \\
\hline Hearing loss and/or vestibular problems & Yes; no & {$[18,21]$} \\
\hline Cancer history & Yes; no & {$[17]$} \\
\hline Parkinson disease & Yes; no & {$[17]$} \\
\hline Alzheimer disease & Yes; no & {$[17]$} \\
\hline Asthma & Yes; no & {$[17]$} \\
\hline Cardiovascular disease & Yes; no & {$[17,18]$} \\
\hline Shortage of breath & No; yes; only if going uphill/hurrying & [14] \\
\hline Problems with headaches & Yes; no & [14] \\
\hline Problems with Walking & No problem; Uses walking aid; Gait problem (no aid); Nonambulant & [23] \\
\hline Sleeping hours & Hours & [14] \\
\hline nocturnal awakenings & Never; often; every night & [14] \\
\hline waking hour overnight & Hours & [14] \\
\hline Number of prescribed drugs and types & $\begin{array}{l}\text { Antidepressants; antipsychotics; antiemetic; } \\
\text { sedatives and hypnotics; medicines for Parkinson's disease; } \\
\text { antihypertensive or antiarrhythmic; analgesics; antiepileptic }\end{array}$ & {$[15-17,21-23]$} \\
\hline
\end{tabular}

intrinsic event (e.g., stroke) or overwhelming hazard; participants were asked to report any fall in the previous year and, consequently, they were classified as fallers or non-fallers for the purposes of the current study. Moreover, after the baseline assessment, participants were contacted by telephone in order to record any falls experienced over a prospective 12-month follow-up.

\section{Data-mining methods}

Three different data-mining approaches were used to develop classifier for faller identification, i.e. the C4.5 decision tree induction algorithm, the Random Forest (RF), and the boosting meta-learning approach AdaboostM1 (AB).

Table 3 Variables related to eye condition and visual function assessed with the structured questionnaire.

\begin{tabular}{ccc}
\hline Variables & Categories or unit of measures & References \\
\hline Ocular conditions & Cataract; pseudophakic; glaucoma; age-related macular degeneration; other retinal degeneration & {$[20,23]$} \\
\hline $\begin{array}{c}\text { Use of bifocal / multifocal } \\
\text { eyeglasses }\end{array}$ & Yes; no & {$[15,23]$} \\
\hline Use of eye drops & Yes; no & Decimals \\
\hline $\begin{array}{c}\text { Best corrected visual acuity in each } \\
\text { eye }\end{array}$ & Decimals \\
\hline Visual acuity loss & Yes; no \\
\hline Recent refraction change & mmHg (average both eyes) \\
\hline Intraocular pressure & Sunny day; rainy day; indifferent \\
\hline Better vision & No; entering indoor; exit indor \\
\hline Blindness effects
\end{tabular}


Table 3 Variables related to eye condition and visual function assessed with the structured questionnaire. (Continued)

$\begin{gathered}\text { Activity of day vision scale: } \\ \text { Items: }\end{gathered}$
Driving at night
Not Applicable (considered as missing data)
Seeing moving objects with night 4 - little difficulty; 3- moderate difficulty; 2- extreme difficulty; 1- unable because
driving
Oncoming headlights
Daytime driving
Drive in unfamiliar areas
Read signs at night
Read signs during the day
See/recognize faces
See television
Read writing on television
Read newspapers
Read medicine bottles
Read food cans
Write checks
Thread a needle

The choice of the algorithm parameters was based on the performances (i.e. accuracy, then sensitivity and finally specificity) estimated by 10 -fold cross-validation: one with $90 \%$ subjects for training and the other with $10 \%$ subjects for validation. Repeating the test 10 times, the classification performance were then calculated by averaging the values obtained from the 10 validation subsets.

C4.5 is the landmark decision tree algorithm developed by Quinlan et al.[25]. The feature of each node is selected in order to divide input samples effectively and information gain is used as a measure of effectiveness. After the induction of the decision tree, a pruning method was applied to reduce the tree's size and complexity.

RF is the state-of-the-art classifier developed by Breiman[26]. It is composed of a number of decision trees that choose their splitting attributes from a random subset of $k$ attributes at each internal node. The best split is taken among these randomly chosen attributes and the trees are built without pruning, as opposed to C4.5. One of the most relevant downsides of using RF, particularly in medical domain data-mining, is that its model is not easily understandable as a single tree. Moreover, we computed the feature importance measures based on Random Forests (RF)[26].

$\mathrm{AB}$ is a meta-learning algorithm which works by incrementally running classifiers on samples of data instances and combining them into an aggregate model[27]. Each individual or weak classifier contributes to the aggregate model in proportion to its accuracy. After each iteration, data instances are reweighted based on incorrect aggregate classifications. This boosts the emphasis of misclassified instances, refining the construction of weak classifiers in future iterations. In the current study, C4.5 was adopted as weak classifier in the $\mathrm{AB}$ algorithm.

$\mathrm{AB}$ classifiers were developed by varying the number of iteration from 20 to 400 and C4.5 trees (both as single classifier and as base classifier in $\mathrm{AB}$ ) were developed by varying confidence factor for pruning from 0.05 to 0.5 , minimum number of instances per leaf from 5 to 20 . MLP were trained by varying the learning rate from 0.3 to 0.9 , the momentum from 0.2 to 1 and the number of epoch form 100 to 2000 . RF was constructed using an ensemble of random trees from 20 to 400 with no depth limit and varying the number of randomly chosen features from $\log _{2}(n)+1$ to $n$, where $n$ is the number of feature. As regards SVM, we used radial basis function kernel, varying gamma from $10^{-5}$ to 10 .

\section{Results}

The study sample consisted of 150 participants (mean age \pm standard deviation: $73.0 \pm 9.6$ years; range: $55-99$ years) including 60 males (40\%) and 90 females (60\%). Participants had a range of severity of visual impairment, for example, BCVA ranging from no light perception to 20/20. 109 participants (72.7\%) suffered from cataract in at least one eye, whereas 42 participants $(28.0 \%)$ were pseudophakic in at least one eye.

The most informative variables, according to the values of feature importance estimated by RF, were: the answer to the item "Read writing on television" of the ADVS, IOP and BCVA in right eye. As shown in Figure 1, among the ten most relevant variables, five were obtained by the ADVS, i.e. the difficulty score, and the following items: "See television"; "Thread a needle"; "Read writing on television"; "Read newspapers".

For each data-mining method, the optimal combination of parameters were selected by maximizing the accuracy estimated by 10 -fold-crossvalidation as shown in Table 4. The ROC curves for identifying fallers are compared in Figure 2. RF and AB outperformed C4.5 in terms of overall accuracy, sensitivity and specificity rates. RF achieved slightly better performances than $\mathrm{AB}$.

Since $A B$ achieved the highest sensitivity, it was interesting to observe the rules obtained from the decision tree 


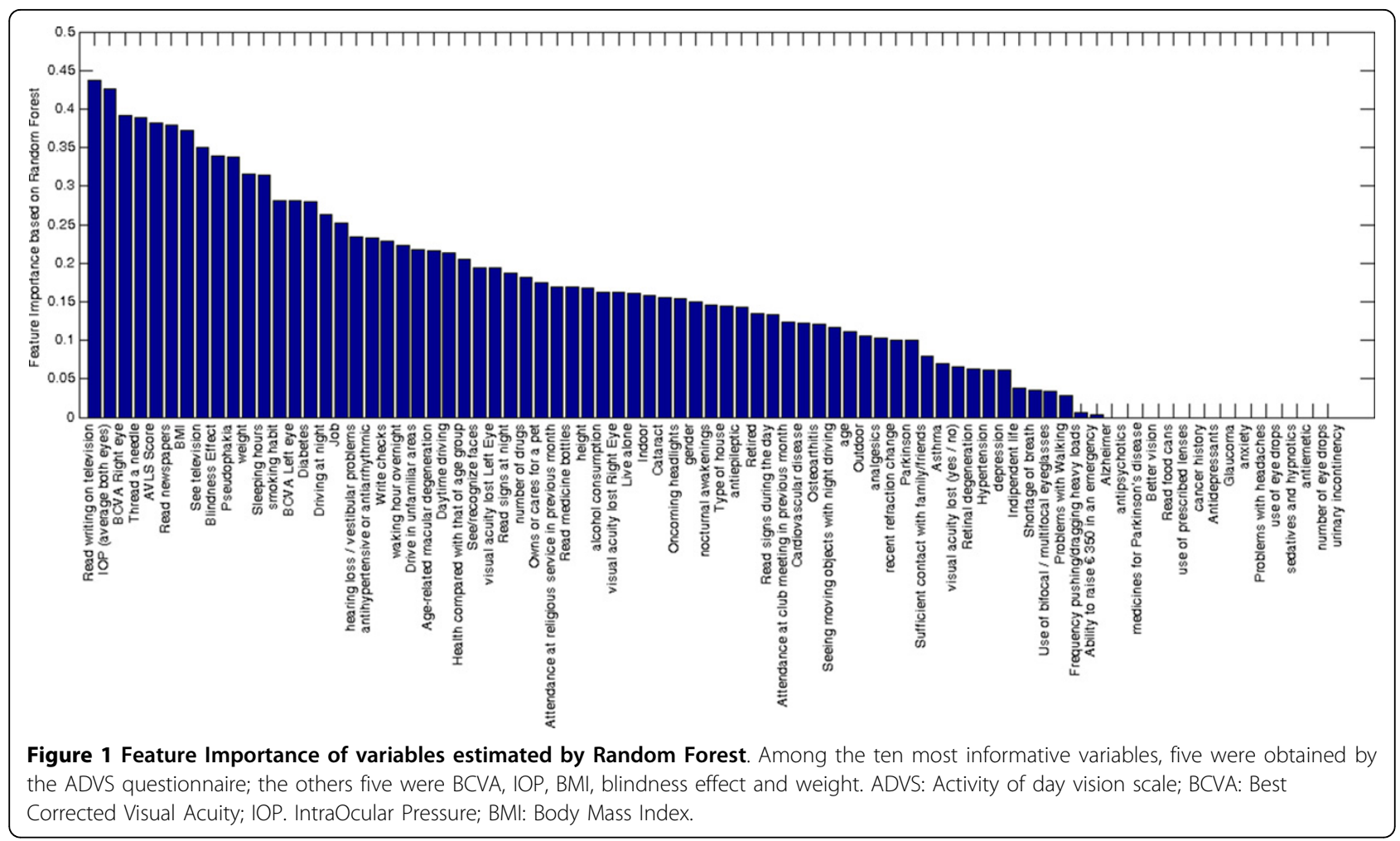

Table 4 Classification performances of the selected classifiers.

\begin{tabular}{|c|c|c|c|c|c|}
\hline Classifier & parameters & $\begin{array}{l}\text { Accuracy } \\
(95 \% \mathrm{Cl})\end{array}$ & Sensitivity & Specificity & AUC \\
\hline$A B$ & NI: 200; CF: 0.5; ML: 25 & $\begin{array}{c}74.7 \% \\
(71.1 \%-77.4 \%)\end{array}$ & $75.8 \%$ & $73.7 \%$ & $82.3 \%$ \\
\hline$C 4.5$ & CF: 0.4; ML: 20 & $\begin{array}{c}63.7 \% \\
(60.0 \%-66.9 \%) \\
\end{array}$ & $57.9 \%$ & $69.5 \%$ & $65.5 \%$ \\
\hline RF & $N T=200 ; N V=15$ & $\begin{array}{c}75.3 \% \\
(71.7 \%-77.9 \%)\end{array}$ & $72.6 \%$ & $77.9 \%$ & $86.2 \%$ \\
\hline
\end{tabular}

$\mathrm{Cl}$ : Confidence Interval;

AB: Adaboost;

RF: Random Forest;

NI: number of iteration;

ML: minimum number of instances per leaf;

CF: confidence factor for pruning;

NT: number of trees;

NF: number of randomly chosen features.

with the highest weights, including or not including ophthalmic features, shown in Figure 3. According to the decision tree including ophthalmic features, the subject was labelled as non-faller if pseudophakic, otherwise, in case of headache problems or IOP higher than 15, the subject was classified as faller. According to the model without any ophthalmic feature (Figure 3a), if the subject referred no or little or moderate difficulties in "Seeing moving objects with night driving", the non-faller label was assigned; otherwise, the classification was based on the presence of anxiety or cardiovascular disease: in case of anxiety and/or any cardiovascular disease, the subject was identified as fallers, whereas the subjects not suffering from anxiety nor any cardiovascular disease were classified as non-fallers.

\section{Discussion}

This paper presented a pilot study to develop a novel tool to identify fallers among ophthalmic patients, based on a few ophthalmological parameters (such as ocular disease, BCVA, IOP) and a standardized questionnaire (including self-evaluation of visual ability). The system 


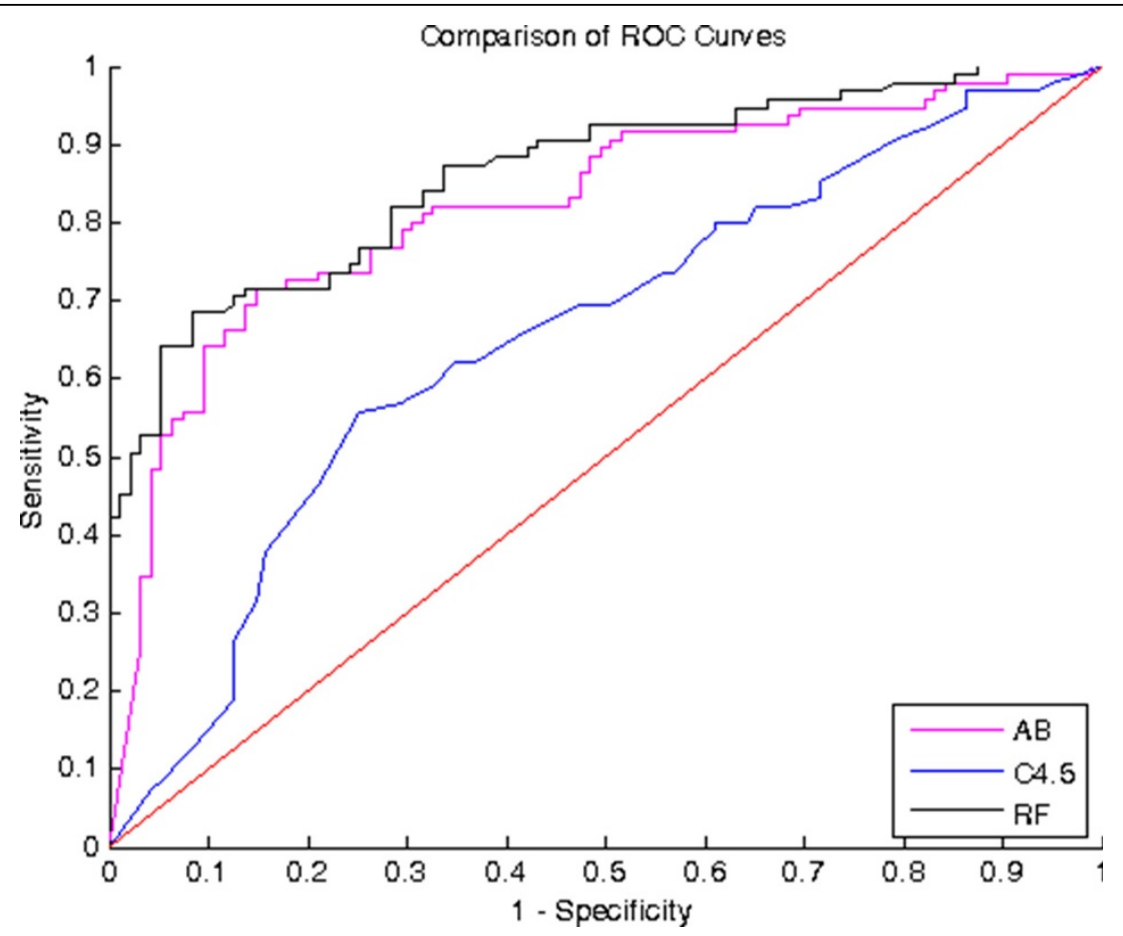

Figure 2 Comparison of the Receiver Operating Curves of the selected classifiers. The RF achieved better performances in terms of Area Under the Curve than C4.5 and AB. RF: Random Forest; AB: AdaBoost.

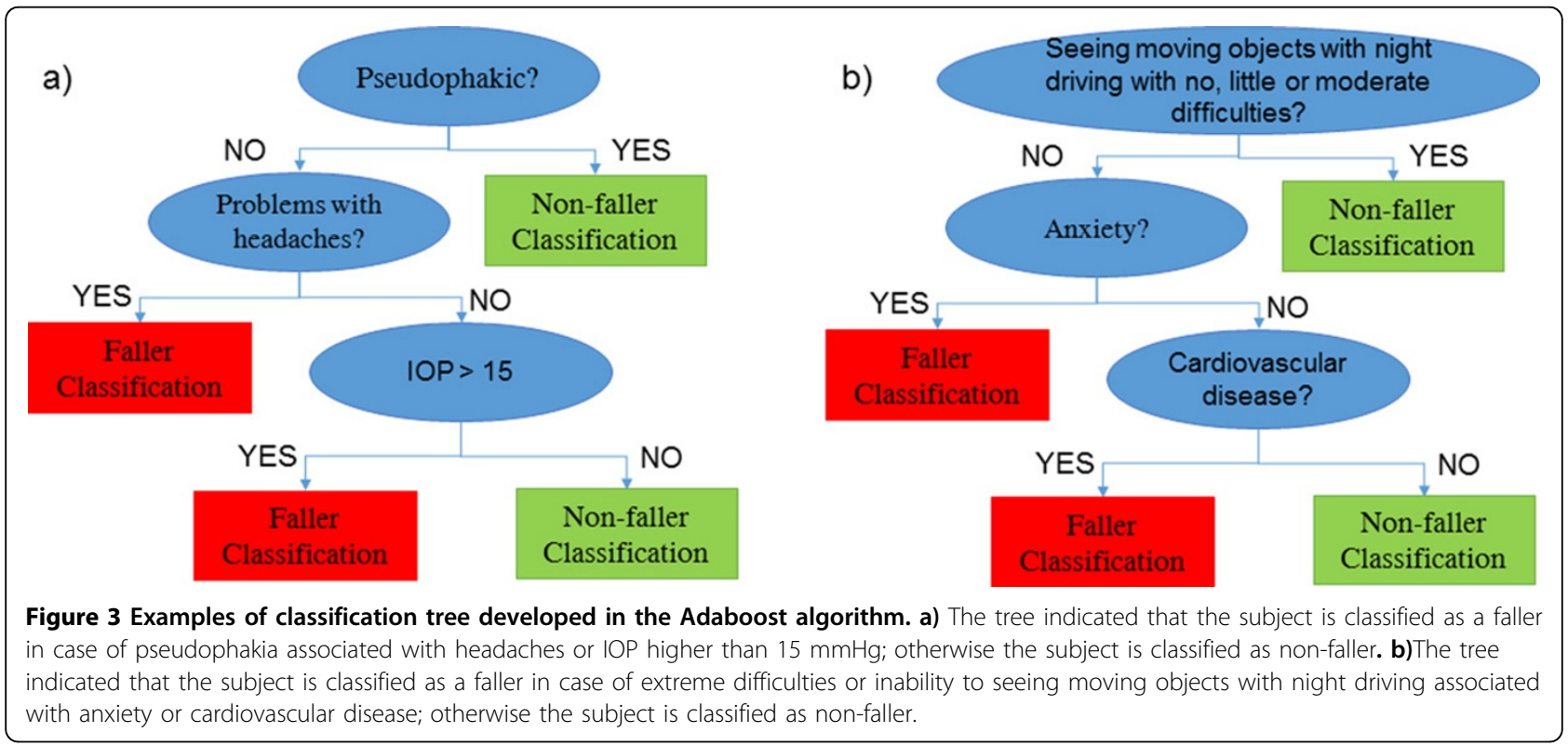

has been realized by comparing different approaches for developing decision tree. Each algorithm achieved a satisfactory performance, e.g. the area under the curve is higher than the performance of random choice (i.e. 0.5) and the Random Forest, the state of art classifier based on decision tree, achieved the best performance, with sensitivity and specificity rate of $72.6 \%$ and $77.9 \%$, respectively.

The comparison between ROC curve of the proposed method and the performance of several functional mobility tests for predicting falls in community-dwelling older people showed that the proposed method achieved 
higher sensitivity and specificity rates than all the functional tests, which had relative risk (RR) ranging from 1.3 to 2.3 and sensitivity and specificity scores ranging from $11 \%$ to $78 \%$, and $28 \%$ to $93 \%[12]$. Moreover, these tests need that the subject could perform a mobility action, for that reason, they could be not suitable for all subjects with visual impairment and, finally, they requires materials and expertise, which are not usually available in an eye clinic. On the contrary, the proposed methods required only few ophthalmological parameters, such as IOP and BCVA, which are routinely measured in eye clinics, and the assessment of a questionnaire, which could filled in part by the physician and in part by the patient (under physician supervision) in about 30 minutes. The developed questionnaire strongly relies on a standardized questionnaire (ADVS), which have been developed for the evaluation of outcome of cataract surgery. We adopted a reduced ADVS version, since it has been shown to have an adequate precision, equivalent criterion validity, improved targeting of item difficulty to patient ability with decreased time for filling the questionnaire[24].

The current study has some limitations, in particular, the small sample size, Therefore, the clinical implications of these findings are potentially relevant, since the requested parameters are based on simple and non-invasive measurements, even if an external and further validation on a large dataset is required. For that reason, a further development of the current study could be the test of a reduced questionnaire including only the most significant variables submitted to a large study sample. Finally, the tool for identification of fallers could be integrated in the webbased platform, developed in the framework of the Smart Health and Artificial Intelligence for Risk estimation (SHARE) project[28]. The platform, now integrated in an open and interoperable cloud computing platform for health and eGovernement (PRISMA), will enable to test the clinical feasibility and uptake of the developed tool in a prospective study. The system has been already tested for cardiovascular disease severity assessment[29] and cardiovascular risk assessment[30]. Moreover, since the most variable could be collected without any specialist expertise, future research will focus on the clinical applicability of the system as a screening tool in non-specialized ambulatories (e.g. at General Practitioners'), in order to identify high-risk patients to be shortlisted for more complex (and costly) investigations. The method could be enhanced by the adoption of instrumental tests[31]. Improved identification of visually impaired individuals at high fall risk may result in more targeted and adequate prevention strategies.

\section{Conclusions}

This study proved that visual assessment and a standardized questionnaire, including the ADVS self-evaluation of visual impairment, could be useful for the automatic identification of fallers among the ophthalmic patients. The developed model enabled to identify fallers among ophthalmic patients with sensitivity and specificity rates of $71.4 \%$ and $87.8 \%$, respectively. These findings pave the way to the development of a novel tool for assessment of fall risk among patient with visual impairment.

\section{Competing interests}

The authors declare that they have no competing interests.

\section{Authors' contributions}

PM analyzed the data and wrote part of the manuscript. AO interpreted the data and wrote part of the manuscript. $\mathrm{AO}$ and MA contributed to the study design. LP contributed to data analysis. FC performed the electronic data collection. SR, FT, and FS conceived of the study, supervised the study and contributed to data interpretation and critically revised the manuscript for important intellectual content. All the authors read and approved the final version of this paper.

\section{Acknowledgements}

The current study was supported by "the 2007-2013 NOP for Research and Competitiveness for the Convergence Regions (Calabria, Campania, Puglia and Sicilia)" with code PON04a3_00139 - Project Smart Health and Artificial intelligence for Risk Estimation.

\section{Declarations}

Publication costs were funded by "the 2007-2013 NOP for Research and Competitiveness for the Convergence Regions (Calabria, Campania, Puglia and Sicilia)" with code PON04a3_00139 - Project Smart Health and Artificial intelligence for Risk Estimation.

This article has been published as part of BMC Medical Informatics and Decision Making Volume 15 Supplement 3, 2015: Multidimensional, multidiscipline and shared decision making in healthcare and eHealth. The full contents of the supplement are available online at http://www. biomedcentral.com/bmcmedinformdecismak/supplements/15/S3.

\section{Authors' details}

${ }^{1}$ SHARE Project, Italian Ministry of Education, Scientific Research and University, Rome, Italy. ${ }^{2}$ Multidisciplinary Department of Medical, Surgical and Dental sciences, Second University of Naples, 80138, Italy. ${ }^{3}$ School of Engineering, University of Warwick, CV47AL, UK.

\section{Published: 11 September 2015}

\section{References}

1. Tinetti ME, Kumar C: The patient who falls: "It's always a trade-off". JAMA 2010, 303(3):258-266

2. Siracuse JJ, Odell DD, Gondek SP, Odom SR, Kasper EM, Hauser CJ, Moorman DW: Health care and socioeconomic impact of falls in the elderly. American journal of surgery 2012, 203(3):335-338, discussion 338

3. Guideline for the prevention of falls in older persons. American Geriatrics Society, British Geriatrics Society, and American Academy of Orthopaedic Surgeons Panel on Falls Prevention. J Am Geriatr Soc 2001 , 49(5):664-672.

4. Peel NM: Epidemiology of falls in older age. Can J Aging 2011, 30(1):7-19.

5. Pecchia L, Bath PA, Pendleton N, Bracale M: Analytic Hierarchy Process (AHP) for examining healthcare professionals' assessments of risk factors. The relative importance of risk factors for falls in communitydwelling older people. Methods Inf Med 2011, 50(5):435-444.

6. Klein BE, Klein R, Lee KE, Cruickshanks KJ: Performance-based and selfassessed measures of visual function as related to history of falls, hip fractures, and measured gait time: the Beaver Dam Eye Study. Ophthalmology 1998, 105(1):160-164.

7. Ivers $R$, Cumming $R$, Mitchell $P$ : Poor vision and risk of falls and fractures in older Australians: the Blue Mountains Eye Study. New South Wales public health bulletin 2002, 13(1-2):8-10.

8. McCarty CA, Fu CL, Taylor HR: Predictors of falls in the Melbourne visual impairment project. Aust N Z J Public Health 2002, 26(2):116-119. 
9. Klein BE, Moss SE, Klein R, Lee KE, Cruickshanks KJ: Associations of visual function with physical outcomes and limitations 5 years later in an older population: the Beaver Dam eye study. Ophthalmology 2003, 110(4):644-650.

10. Legood R, Scuffham P, Cryer C: Are we blind to injuries in the visually impaired? A review of the literature. Inj Prev 2002, 8(2):155-160.

11. Perell KL, Nelson A, Goldman RL, Luther SL, Prieto-Lewis N, Rubenstein LZ: Fall risk assessment measures: an analytic review. J Gerontol A Biol Sci Med Sci 2001, 56(12):M761-766.

12. Tiedemann A, Shimada H, Sherrington C, Murray S, Lord S: The comparative ability of eight functional mobility tests for predicting falls in community-dwelling older people. Age Ageing 2008, 37(4):430-435.

13. Ahmad R, Bath PA: The use of Cox regression and genetic algorithm (CoRGA) for identifying risk factors for mortality in older people. Health Informatics Journal 2004, 10(3):221-236.

14. Bath PA, Pendleton N, Morgan K, Clague JE, Horan MA, Lucas SB: New approach to risk determination: Development of risk profile for new falls among community-dwelling older people by use of a genetic algorithm neural network (GANN). J Gerontol a-Biol 2000, 55(1):M17-M21.

15. Coleman AL, Stone K, Ewing SK, Nevitt M, Cummings S, Cauley JA, Ensrud KE, Harris EL, Hochberg MC, Mangione CM: Higher risk of multiple falls among elderly women who lose visual acuity. Ophthalmology 2004, 111(5):857-862.

16. de Boer MR, Pluijm SM, Lips P, Moll AC, Völker-Dieben HJ, Deeg DJ, van Rens GH: Different aspects of visual impairment as risk factors for falls and fractures in older men and women. J Bone Miner Res 2004, 19(9):1539-1547.

17. Knudtson $M D$, Klein $B E$, Klein R: Biomarkers of aging and falling: the Beaver Dam eye study. Arch Gerontol Geriatr 2009, 49(1):22-26.

18. Kulmala J, Era P, Parssinen O, Sakari R, Sipila S, Rantanen T, Heikkinen E: Lowered vision as a risk factor for injurious accidents in older people. Aging Clin Exp Res 2008, 20(1):25-30

19. Lord SR, Ward JA, Williams P, Anstey KJ: Physiological factors associated with falls in older community-dwelling women. Journal of the American Geriatrics Society 1994, 42(10):1110-1117.

20. Wood JM, Lacherez P, Black AA, Cole MH, Boon MY, Kerr GK: Risk of falls, injurious falls, and other injuries resulting from visual impairment among older adults with age-related macular degeneration. Invest Ophthalmol Vis Sci 2011, 52(8):5088-5092.

21. Bongue B, Dupre C, Beauchet O, Rossat A, Fantino B, Colvez A: A screening tool with five risk factors was developed for fall-risk prediction in community-dwelling elderly. J Clin Epidemiol 2011, 64(10):1152-1160.

22. Tromp AM, Pluijm SM, Smit JH, Deeg DJ, Bouter LM, Lips P: Fall-risk screening test: a prospective study on predictors for falls in communitydwelling elderly. J Clin Epidemiol 2001, 54(8):837-844.

23. Lord SR, Dayhew J, Howland A: Multifocal glasses impair edge-contrast sensitivity and depth perception and increase the risk of falls in older people. J Am Geriatr Soc 2002, 50(11):1760-1766.

24. Pesudovs K, Garamendi E, Keeves JP, Elliott DB: The Activities of Daily Vision Scale for cataract surgery outcomes: re-evaluating validity with Rasch analysis. Invest Ophthalmol Vis Sci 2003, 44(7):2892-2899.

25. Quinlan JR: C4.5 : programs for machine learning San Mateo, Calif:. Morgan Kaufmann Publishers; 1993.

26. Breiman L: Random Forests. Mach Learn 2001, 45(1):5-32

27. Freund $Y$, Schapire RE: Experiments with a new boosting algorithm. ICML: 1996 1996, 148-156.

28. Melillo P, Scala P, Crispino F, Pecchia L: Cloud-Based Remote Processing and Data-Mining Platform for Automatic Risk Assessment in Hypertensive Patients. Ambient Assisted Living and Daily Activities Springer; 2014, 155-162.

29. Melillo P, De Luca N, Bracale M, Pecchia L: Classification Tree for Risk Assessment in Patients Suffering From Congestive Heart Failure via LongTerm Heart Rate Variability. IEEE J Biomed Health Inform 2013, 17(3):727-733.

30. Melillo P, Izzo R, Orrico A, Scala P, Attanasio M, Mirra M, De Luca N, Pecchia L: Automatic Prediction of Cardiovascular and Cerebrovascular Events Using Heart Rate Variability Analysis. PLOS ONE 2015, 10(3):e0118504.

31. Melillo P, Pecchia L, Testa F, Rossi S, Bennett J, Simonelli F: Pupillometric analysis for assessment of gene therapy in Leber Congenital Amaurosis patients. Biomed Eng Online 2012, 11(1):40.

doi:10.1186/1472-6947-15-S3-S6

Cite this article as: Melillo et al:: A pilot study for development of a novel tool for clinical decision making to identify fallers among ophthalmic patients. BMC Medical Informatics and Decision Making 2015 15(Suppl 3):S6.

\section{Submit your next manuscript to BioMed Central and take full advantage of:}

- Convenient online submission

- Thorough peer review

- No space constraints or color figure charges

- Immediate publication on acceptance

- Inclusion in PubMed, CAS, Scopus and Google Scholar

- Research which is freely available for redistribution

Submit your manuscript at www.biomedcentral.com/submit
Biomed Central 